\title{
Retour d'expérience des attentats du 13 novembre 2015. La régulation médicale zonale
}

\author{
Feedback on Terrorist Attacks on November 13, 2015. The Medical Zonal Regulation
}

\author{
M. Nahon $\cdot$ N. Poirot $\cdot$ JS Marx $\cdot$ M. Lejay $\cdot$ S. Tartière $\cdot$ C. Chastre $\cdot$ C. Fuilla $\cdot$ P. Pelloux $\cdot$ B. Vivien $\cdot$ C. Télion $\cdot$ \\ P. Carli
}

Reçu le 9 janvier 2016; accepté le 11 janvier 2016

(C) SFMU et Lavoisier SAS 2016

Résumé Pour faire face à l'attentat terroriste multisite du 13 novembre 2015, une régulation médicale zonale des huit Samu de la région Île-de-France a été mise en place au Samu de Paris. Elle a permis, en utilisant une stratégie de sectorisation des moyens Smur et des ressources hospitalières, la médicalisation des sites, la régulation et le transport par des équipes médicales de la majorité des victimes en urgence absolue. Des moyens Smur et des hôpitaux ont pu être gardés en réserve pour faire face à une aggravation de la situation (notamment sur le site du Bataclan) et à d'éventuelles autres attaques. L'analyse de cette expérience montre de nombreux points forts mais aussi des pistes d'amélioration utiles pour tous ceux qui seraient confrontés à un tel événement.

Mots clés Attentats terroristes multisite - Samu - Smur · Mobilisation · Organisation - Sectorisation · Triage · Damage control $\cdot$ Plan Blanc $\cdot$ Plan Camembert Abstract To face the multisite terrorist attacks on November
13, 2015, a medical zonal regulation system was set up at the
Samu de Paris in order to coordinate the 8 Samu of the Île-

M. Nahon $\cdot$ N. Poirot $\cdot$ JS Marx $\cdot$ M. Lejay $\cdot$ S. Tartière

C. Chastre $\cdot$ C. Fuilla $\cdot$ P. Pelloux $\cdot$ B. Vivien $\cdot$ C. Télion ·

P. Carli $(\bowtie)$

Assistance Publique - Hôpitaux de Paris,

Centre Hospitalier Universitaire Necker - Enfants Malades,

Samu de Paris, service d'anesthésie-réanimation,

149 rue de Sèvres, F-75730 Paris cedex 15

e-mail : pierre.carli@aphp.fr

C. Fuilla

Direction Centrale du Service de Santé des Armées,

60 boulevard du Général Marcel Valin, F-75509 Paris cedex 15

B. Vivien · P. Carli

Université Paris Descartes - Paris V,

12 rue de l'École de Médecine, F-75270 Paris cedex 06
de-France region. This system, by using a sectorization strategy of the medical means (mobile intensive care unit [MICU]) and hospital resources, enabled on site medicalization, regulation and transportation by medical teams of most of the patients presenting immediate emergencies. MICU and hospitals were kept available in case the situation had worsened (especially on the Bataclan site) or other attacks had occurred. The analysis of this experience shows many strong points but also useful areas of improvement for all those who might face such event.

Keywords Multisite terrorist attacks $\cdot$ Samu $\cdot$ Smur . Mobilization · Organization - Sectorization · Triage · Damage control $\cdot$ White plan $\cdot$ Camembert plan

\section{Introduction}

L'attentat multisite du vendredi 13 novembre 2015 à Paris est l'événement terroriste le plus grave qui se soit produit en France depuis l'existence du Samu, et le plus complexe que les services d'urgences aient eu à gérer [1]. Très différent des attentats de 1995 et 1996 à Paris, à la fois par la violence et par sa dimension [2], cet événement n'a pas été une totale surprise. Depuis 2006, à la suite notamment des attentats terroristes de Madrid (2004) et de Londres (2005) puis de Bombay (2008), une organisation spécifique des Samu et des hôpitaux avait été planifiée. Les grandes lignes de ce plan «Attentat multisite » surnommé « plan Camembert » précisent :

- la nécessité d'éviter l'accumulation des moyens médicaux sur le premier site, grâce à un engagement raisonné des moyens. Un marquage au fur et à mesure de l'apparition de chaque site est effectué par une unité mobile hospitalière $(\mathrm{UMH})$ de proximité et un médecin régulateur en association avec le dispositif de la Brigade de sapeurspompiers de Paris (BSPP) sous le commandement d'un 
commandant des opérations de secours (COS) et d'un directeur des secours médicaux (DSM) ;

- l'activation, dès l'apparition du $2^{\mathrm{e}}$ site, d'une régulation zonale des 8 Samu d'Île-de-France et la mise en œuvre d'un découpage de Paris et de l'Île-de-France en trois secteurs (ou parts de « Camembert »).

Cette sectorisation permet l'organisation des renforts Smur sur les sites : les Smur des départements de la petite couronne renforcent immédiatement Paris, ceux de la grande couronne se rapprochent et se positionnent dans la petite couronne (Fig. 1). L'hospitalisation des victimes se fait dans le même secteur de façon centrifuge en réservant les plateaux techniques lourds de Paris intramuros aux blessés les plus graves.

Initialement prévu pour des blessés par explosions de bombes artisanales, ce dispositif avait été récemment adapté aux principes du « Damage Control » pour les blessés par balles présentant des hémorragies actives [3].

Jusqu'au vendredi 13 novembre 2015, ce plan n'avait jamais été utilisé réellement mais avait donné lieu à des exercices, le dernier en date s'étant déroulé ce même vendredi 13 novembre 2015 à $9 \mathrm{~h} 30$ à la régulation du Samu zonal et à l'État-major de la BSPP. Le scénario de cet exercice, jugé par les participants comme un peu excessif, envisageait un attentat provoqué par quatre commandos sur dix sites avec un bilan de 66 décédés, 74 urgences absolues (UA) et 48 urgences relatives (UR). Le soir même, la réalité allait dépasser la fiction...

\section{La régulation médicale au Samu de Paris}

\section{L'alerte}

Vers $21 \mathrm{~h} 00$ au Samu de Paris, le vendredi 13 novembre 2015, la situation est calme, et le dispositif de garde est en place : deux médecins régulateurs Samu, quatre assistants de régulation médicale (ARM), deux médecins régulateurs de la Permanence des soins ambulatoires (PDSA) et deux régulateurs pour les secouristes (Protection Civile). Tous les Smur de Paris sauf un sont à leur base.

À $21 \mathrm{~h} 25$, le Samu 93 signale une explosion au Stade de France. Celle-ci est confirmée par les associations de secouristes 2 minutes plus tard. À 21h27, de nombreux appels de témoins alertent directement le Samu de Paris pour une fusillade rue Bichat avec de nombreux morts et blessés. L'attentat multisite est immédiatement identifié et dans les minutes qui suivent, la BSPP demande le déclenchement du plan « nombreuses victimes » (plan NOVI), tandis que l'Assistance Publique-Hôpitaux de Paris (AP-HP) déclenche le Plan Blanc. L'ensemble des Samu d'Île-de-France est prévenu et la répartition des Smur s'organise pour les envoyer sur les différents sites. Pour respecter la stratégie du « Camembert », aucune équipe de Paris n'est envoyée au Stade de France, et le Samu 93 est renforcé par le Samu 92 et le Samu 95 (Fig. 1).

L'ensemble des personnels du Samu de Paris et des Smur de Necker sont rappelés. La régulation médicale est immédiatement renforcée par les médecins du service présents à

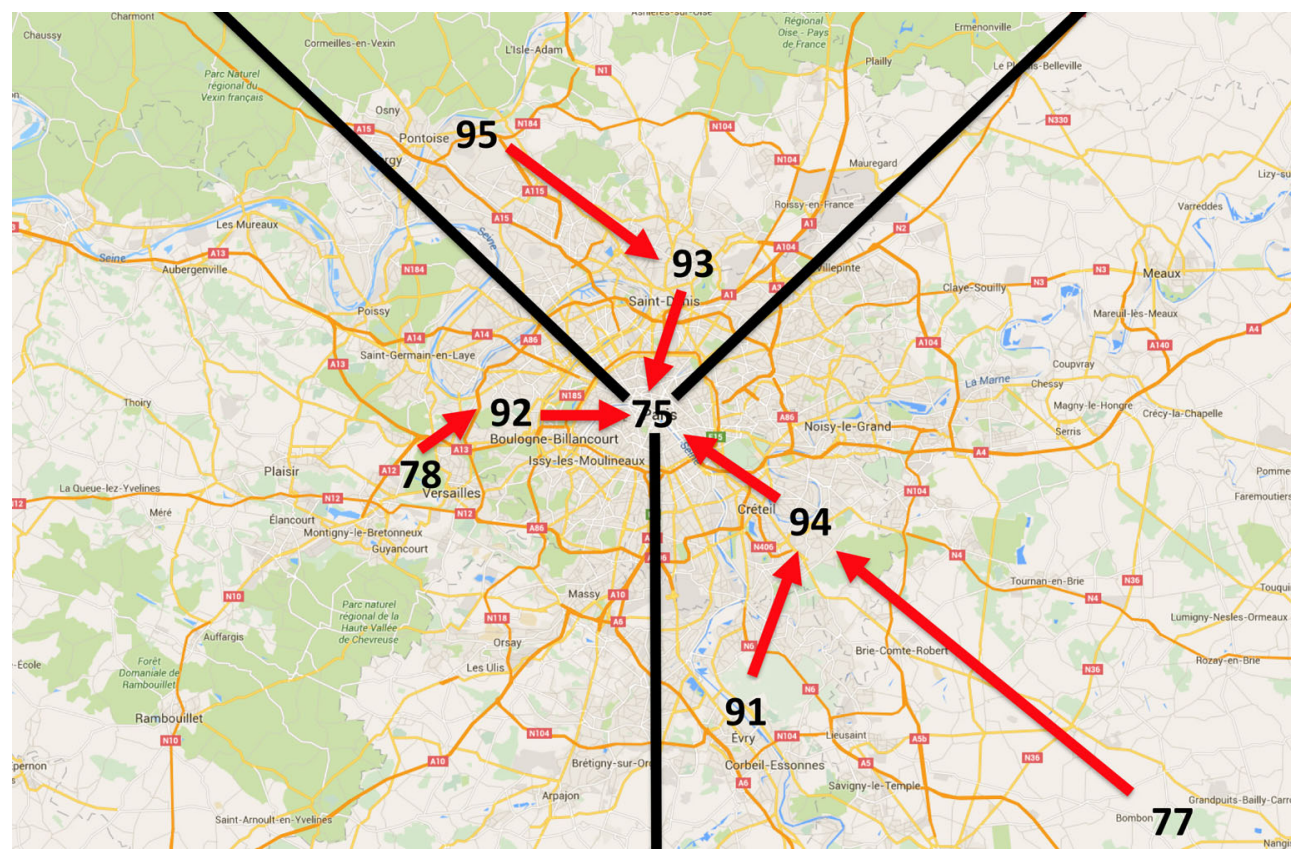

Fig. 1 Sectorisation des renforts Smur zonaux pour la stratégie du « Camembert ». Les numéros désignent les Samu départementaux de rattachement 
l'hôpital, de garde en anesthésie et en réanimation. Au même moment, survient un pic d'appel au 15, confirmant la notion de sites multiples, avec identification d'un nouveau site au niveau du Bataclan. La salle de crise zonale est activée avec les personnels rappelés. Plusieurs équipes Smur sont composées et armées dès l'arrivée des personnels rappelés. La régulation du Samu continue à gérer le quotidien. Des moyens Smur sont conservés pour intervenir sur les pathologies « du quotidien » : ainsi, plusieurs appels pour douleurs thoraciques sont pris en charge sans retard dans la soirée.

À 21h45, la régulation médicale du Samu de Paris est en position de régulation zonale des huit Samu d'Île-de-France. Ses missions imposent donc de :

- diviser la capitale en trois secteurs : le secteur Nord géré par le Samu 93 (Stade de France), le secteur Est (fusillades et Bataclan), le secteur Ouest, sans attentat pour l'instant, mais gardé en réserve en cas d'apparition d'un nouveau site (Fig. 2) ;

- répartir les Smur recrutés par chaque Samu sur les sites en une première vague (marquage de chaque site), puis en fonction des premiers bilans reçus, en une deuxième vague de renfort. Quarante-cinq équipes Smur sont répar- ties sur le terrain et 25 autres sont maintenues en réserve, ou affectées à l'activité quotidienne ;

- alerter l'ensemble des salles de réveils susceptibles de recevoir en admission directe, dans le cadre du Plan Blanc, des victimes en urgence absolue (UA). Les disponibilités des blocs opératoires et des services de réanimation de chaque site sont affichées en salle de crise zonale ;

- fournir aux médecins régulateurs des différents sites d'attentats, les capacités des différents hôpitaux receveurs en termes de flux ;

- prévenir les hôpitaux de l'arrivée des victimes en fonction des informations transmises depuis les différents sites.

\section{L'organisation de la salle de crise}

Plus de 22 personnes (médecins, ARM, secrétaires) ont participé au fonctionnement de la régulation médicale zonale. L'ouverture de la salle de crise avec peu de personnel a très vite conduit à abandonner l'informatique, des tableaux blancs et une carte de situation murale se révélant rapidement être les moyens les plus efficaces pour suivre et afficher l'évolution des sites. La gestion des communications avec les autres

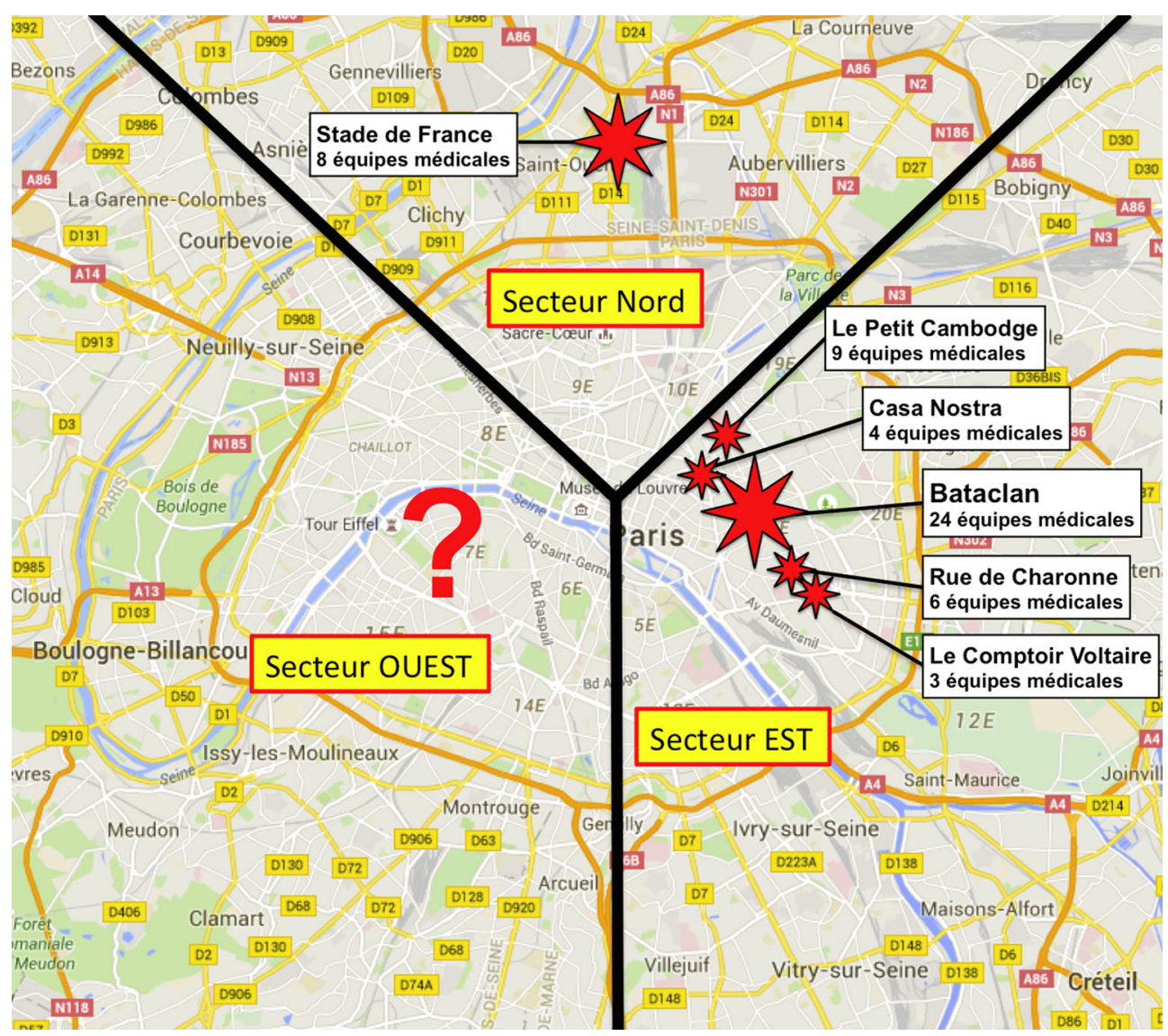

Fig. 2 Sectorisation de Paris en « Camembert» et moyens médicaux 


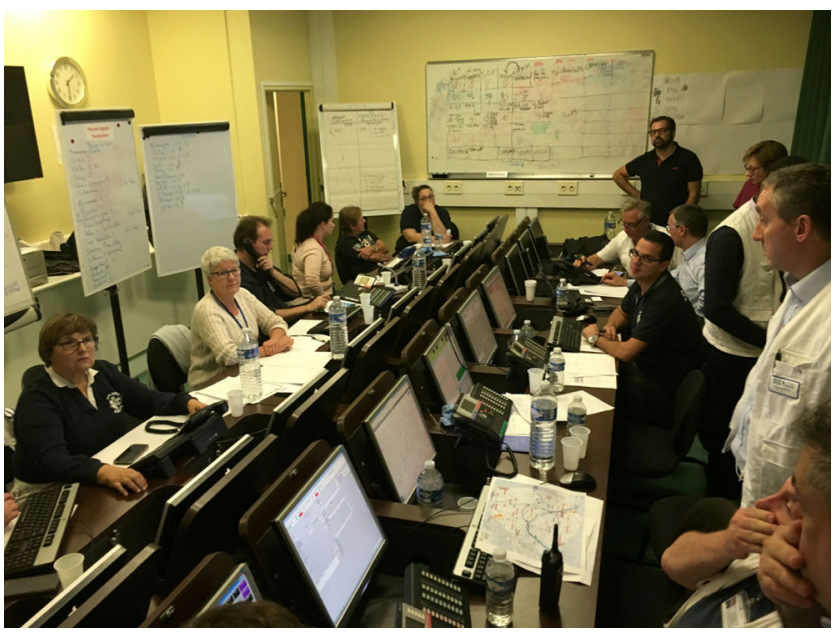

Fig. 3 La salle de crise de la régulation du Samu zonal vers 22h30

services, Préfecture de Police, et État-major de la BSPP reste complexe. Des initiatives pragmatiques voient le jour : la régulation zonale s'organise en secteurs, chaque régulateur Samu sur le site s'adressant à un correspondant identifié qui retranscrit ses informations sur les tableaux et les cartes. Un médecin coordonne le recueil de l'ensemble des informations. Un représentant de l'Agence Régionale de Santé (ARS) assure la liaison entre les différents services. Toutes les communications auraient dû passer par le dispositif radio ANTARES. En pratique, les GSM personnels et ceux du service ont été utilisés pour les communications. Des SMS ont été envoyés, indiquant les places disponibles et les hôpitaux d'accueil.

\section{Le maintien d'une réserve pour un potentiel d'aggravation}

Le principe du plan attentats multisite est d'anticiper une couverture complète du territoire pour garder des moyens d'intervention et d'hospitalisation dans toute la capitale. Les attentats du vendredi 13 novembre 2015 ont été localisés dans les secteurs Nord et Est, respectant le secteur Ouest. Ce secteur a donc été gardé en réserve. Les moyens Smur du secteur Ouest n'ont été utilisés qu'en fin de dispositif et les hôpitaux de ce même secteur n'ont pas ou peu reçu de blessés en lien avec les attentats. Il faut noter que l'enquête de Police a montré a posteriori qu'un attentat supplémentaire était prévu dans le secteur Nord, ainsi que dès le lendemain une attaque de centre commercial dans le secteur Ouest.

\section{La régulation médicale sur les sites}

Pour chacun des sites, un médecin régulateur Samu aurait dû être affecté à chaque PMA mis en place.

En pratique, deux types de situations ont été rencontrés et ont considérablement influencé la régulation médicale.

\section{Sites de fusillades}

Les deux principaux sites sont la rue Bichat et la rue de Charonne. Sur ces sites, il n'a pas été constitué réellement de PMA fixe. En pratique, un point de rassemblement des victimes (PRV) médicalisé s'est révélé plus simple. Un trio, constitué d'un officier de la BSPP, d'un médecin chef de la BSPP et d'un régulateur du Samu, a géré le site. Le médecin régulateur du Samu a affecté les blessés dans les hôpitaux en fonction des informations de disponibilité de places hospitalières transmises par la régulation zonale. En l'absence d'information et lorsque le principe du « damage control » rendait nécessaire une évacuation immédiate, les victimes ont été directement dirigées vers les hôpitaux du secteur de « Camembert » adaptés à l'état de la victime. Sur ces sites, la sécurisation des abords et de l'itinéraire a conduit à des difficultés d'évacuation. Des décisions d'évacuation par brancardage à pieds ont été prises afin d'éviter une perte de temps pour la réalisation de l'hémostase chirurgicale des lésions hémorragiques.

Ainsi les hôpitaux Saint-Louis (à quelques dizaines de mètres de la rue Bichat) et Saint-Antoine (très proche de la rue de Charonne) ont reçu des UA en évacuations de proximité. Le schéma classique de régulation a donc été contourné dans deux circonstances :

- arrivée directe des blessés transportés par des témoins sans régulation ;

- évacuation de proximité régulée par le site, avec information rétroactive de la régulation zonale.

On peut estimer que $25 \%$ des victimes sont rentrées dans ce cadre. Le Tableau 1 résume la répartition définitive des UA et des urgences relatives (UR) dans les hôpitaux, quelles que soient les modalités de leur admission.

\section{Le site du Bataclan}

La situation sur ce site est très différente des sites de fusillades en pleine rue. En effet, à la fusillade sont associés :

- des explosions de kamikazes en milieu clos, à l'origine de multiples victimes présentant des traumatismes pénétrants et éventuellement des lésions de blast ;

- une prise d'otages en zone de combat retardant l'évacuation de blessés, dont certains en état critique. Certaines victimes se sont réfugiées à proximité immédiate du site dans des halls d'immeubles, constituant des « nids» de blessés, d'accès et d'extraction difficiles ;

- un potentiel évolutif imprévisible. Plusieurs centaines de spectateurs pris en otages pouvaient faire craindre non seulement une hécatombe, mais un nombre de blessés très important et très supérieur à celui des autres sites (estimé sur le terrain à 100 UA supplémentaires). 


\begin{tabular}{|lllll|}
\hline Tableau $\mathbf{1}$ Moyens Samu et Smur mobilisés sur les différents sites. & & \\
\hline Ordre chronologique & $\mathbf{1}$ & $\mathbf{2}$ & $\mathbf{3}$ & $\mathbf{4}$ \\
\hline Site & Stade de France & Rue Bichat & Bataclan & Rue de Charonne \\
Samu régulateur & 93 & 75 & 75 & 75 \\
Nombre d'UMH & 8 & 9 & 24 & 6 \\
Nombre d'UA & 6 & 16 & 26 & 7 \\
Nombre d'UR & $>30$ & 12 & 50 & 32 \\
\hline UMH : unité mobile hospitalière ; UA : urgence absolue ; UR : urgence relative. & & \\
\end{tabular}

La durée de l'intervention sur ce site a donc largement dépassé celle des autres. En conséquence, une chaîne médicale plus classique que pour les fusillades a été mise en place [4]. Un double poste médical avancé (PMA) pour les UA et pour les UR a été installé à $200 \mathrm{~m}$ du Bataclan. Il a permis la prise en charge des victimes au fur et à mesure de leur évacuation depuis les « nids» de blessés situés à proximité immédiate de la salle de spectacle. Malgré la sécurisation du site du PMA par la Police, plusieurs alertes (intrusion d'un véhicule non identifié, tirs à proximité) se sont produites. La médicalisation de ce site a été considérablement renforcée par la mobilisation zonale, permettant la prise en charge de toutes les UA par une équipe médicale de Smur ou d'une AR de la BSPP.

\section{Quelques initiatives de terrain intéressantes}

- L'évacuation sécurisée des victimes a été réalisée par groupes de patients, sous escorte policière ou militaire, vers une destination unique. Pour éviter un afflux sur les hôpitaux receveurs, des groupes mixtes ont été constitués avec 2 ou 3 UA et 2 ou 3 UR dans 4 à 5 véhicules maximum. Cette organisation a remplacé les « colonnes » de victimes (UA ou UR) envisagées dans le plan ;

- des médecins étaient présents sur place, en tant que témoins ou voisins de sites d'attentats, et en particulier certains médecins de Smur. Lorsqu'ils se sont présentés aux médecins régulateurs des sites, ils ont été directement intégrés au dispositif. D'autres médecins ont aidé les secours en donnant une alerte précise depuis le site où ils se trouvaient ;

- la constitution d'équipes Smur supplémentaires a pu être effectuée grâce au rappel des personnels Samu-Smur, ce qui a permis de disposer de plus d'équipes médicales que de véhicules disponibles. Certaines de ces équipes ont été envoyées sur les sites simultanément dans un même vecteur. Elles ont ensuite médicalisé des véhicules de secouristes, prenant part aux évacuations groupées. Une réflexion sur le matériel dont pourraient être dotées ces équipes Smur supplémentaires est nécessaire ;
- enfin, l'anticipation de l'aggravation du bilan sur le site du Bataclan a été à l'origine d'une mobilisation de moyens dépassant la zone de défense Île-de-France. La régulation zonale du Samu, en contact avec la Direction Générale de la Santé et le Service de Santé des Armées, a été à l'origine de la mobilisation de neuf hélicoptères médicalisés, d'équipes Smur terrestres et de possibilités d'hospitalisation qui seront envisagées dans un autre paragraphe.

\section{Discussion}

\section{Points forts}

L'analyse du fonctionnement de la régulation médicale, en confrontant ce qui était théoriquement prévu à la réalité du terrain, permet de confirmer certaines options et de proposer des pistes d'amélioration. Mais de nombreux points qui avaient été anticipés se sont révélés parfaitement efficaces, ont donné satisfaction et expliquent le bon fonctionnement global du dispositif. Ainsi, la mobilisation coordonnée des Samu, l'engagement raisonné des renforts et plus généralement la stratégie du « camembert » se sont révélés adaptés. De même, l'organisation de plusieurs niveaux de régulation sur le terrain, au niveau départemental et zonal, a bien fonctionné. Le maintien de moyens en réserve (extra- et intrahospitaliers) a aussi participé à la maîtrise d'un événement évolutif. Enfin, la coopération Samu-Pompiers aussi bien sur site qu'entre la régulation du Samu de Paris et la Coordination médicale de la BSPP, a été particulièrement simple et efficace. La simplification des procédures de régulation a aussi permis avec pragmatisme de réaliser des évacuations et des prises en charge compatibles avec les principes du « damage control », confirmant ainsi l'organisation des soins mise en ouvre au cours des attentats de «Charlie Hebdo » en janvier 2015 [5]. Enfin, les principes de la médecine de catastrophe ont été utilisés sans dogmatisme et avec adaptabilité. Ainsi, un PMA a été mis en place quand cela était nécessaire, mais a été remplacé pour gagner du temps par une organisation plus légère quand cela était possible. 


\section{Pistes d'amélioration}

Un système basé sur l'échange d'informations avec le terrain est particulièrement tributaire de la maîtrise des communications. Cependant, les moyens de communications comme le réseau ANTARES n'ont pas donné entière satisfaction, pour des raisons tenant à la fois de facteurs liés au système et à son utilisation. Le réseau GSM a donc été largement utilisé malgré les risques de coupure qui aurait pu être décidée par les autorités pour raison de sécurité, ou plus simplement de saturation du réseau. Il était prévu qu'en cas de coupure de communication, chaque médecin régulateur sur site oriente les patients dans son secteur de «Camembert », en informant seulement a posteriori la régulation zonale. Quelques évacuations ont été réalisées suivant ce principe. Enfin, pour améliorer la communication entre les salles de crise, il est envisagé qu'un médecin du Samu rejoigne la salle de crise de la BSPP, et qu'inversement un médecin de la BSPP s'intègre à celle du Samu. Il faut noter que la difficulté de maîtriser les communications a été signalée comme un point d'amélioration dans d'autres cas d'attentats par fusillades [6].

Contrairement à ce qui avait été réalisé à l'exercice, l'informatique n'a pas été utilisée directement pour gérer l'événement. La possibilité d'incrémenter un site Web partagé entre tous les acteurs constitue un moyen simple d'informer l'ensemble des équipes mobilisées de l'évolution de la situation. Elle apporte une vision globale aux acteurs, y compris ceux qui attendent d'être engagés sur le terrain ou à l'hôpital. Cette amélioration est en cours de réalisation. De même, il est envisagé qu'un réseau radiocomplémentaire permette si besoin de joindre simultanément toutes les équipes Smur d'Île-de-France ainsi que l'ensemble des hôpitaux, leur permettant de suivre l'évolution sur le terrain et de se préparer en conséquence.

Si les modalités de déclenchement du Plan Blanc et du plan attentats multisite semblent pertinentes, la levée du dispositif n'est en revanche pas bien codifiée. Le 14 novembre 2015 à 4h30, le dispositif préhospitalier est clôturé, mais le Plan Blanc reste en vigueur, en raison de l'incertitude planant sur le risque de survenue d'autres attentats. Pour les équipes maintenues en réserve, cette incertitude est encore plus difficile à gérer : jusqu'à quand maintenir la mobilisation ou à quel moment faut-il renforcer les sites qui ont pris en charge les victimes s'il n'y a pas d'autres attentats immédiats?

Il a également fallu gérer les relèves et le remplacement des équipes mobilisées qui étaient prévues de garde le lendemain samedi 14 novembre. Ainsi, dès la fin de la prise d'otage au Bataclan, certaines équipes restées en réserve ont été renvoyées à leur domicile, et affectées au planning pour assurer la garde du samedi.

Pendant la nuit du vendredi 13 au samedi 14 novembre, mais aussi le lendemain, les fausses alertes à la régulation du Samu ont représenté un véritable problème. Dans la soirée du vendredi 13 novembre, plusieurs autres sites d'attentats ont été signalés en même temps que les fusillades du $\mathrm{X}^{\mathrm{e}}$ et $\mathrm{XI}^{\mathrm{e}}$ arrondissements. Ces sites n'ont pu être infirmés qu'après une vérification physique par la Police ou les Pompiers, mobilisant ainsi des moyens qui auraient été plus utiles ailleurs. Le lendemain, samedi 14 novembre, deux fausses alertes particulièrement précises ont nécessité l'envoi de moyens de secours et même d'équipes de Smur. Un objectif mal intentionné de maintenir les équipes sous pression ou d'observer le comportement des secours ne peut pas être exclu.

\section{Conclusion}

L'attentat terroriste du 13 novembre 2015 est le premier attentat multisite survenu dans un pays disposant d'un système de soins préhospitaliers médicalisés et d'une régulation médicale. L'organisation de cette régulation à un niveau zonal regroupant les huit Samu de la région a permis la mise en œuvre d'une stratégie sectorielle de répartition des moyens Smur et d'évacuation vers les ressources hospitalières, selon la stratégie dite du « camembert ». Cette stratégie s'est révélée efficace face à une situation complexe et évolutive. Elle a rendu possible la couverture médicale des différents sites et l'anticipation d'une aggravation de la situation, tout en maintenant des moyens Smur et des hôpitaux en réserve. Les principes du « Damage control » préhospitalier pour les UA victimes de fusillades ont été confortés par la régulation médicale, en favorisant un accès rapide aux blocs opératoires des hôpitaux de référence ou de proximité. Enfin, en permettant une organisation efficace des soins et en maintenant leur qualité, la régulation médicale s'est opposée directement aux objectifs du terrorisme.

Liens d'intérêts : Les auteurs déclarent ne pas avoir de lien d'intérêt.

\section{Références}

1. Hirsh M, Carli P, Nizard R, et al (2015) The medical response to multisite terrorist attacks in Paris. Lancet 386:2535-8

2. Carli P, Telion C, Baker D (2003) Terrorism in France. Prehosp Disaster Med 18:92-9

3. Tourtier JP, Palmier B, Tazarourte K, et al (2013) The concept of damage control: extending the paradigm in the prehospital setting. Ann Fr Anesth Reanim 32:520-6

4. Baker DJ, Télion C, Carli P (2007) Multiple casualty incidents: the prehospital role of the anesthesiologist in Europe. Anesthesiol Clin 25:179-88

5. Tourtier JP, Pelloux P, Dang Linh P, et al (2015) Charlie Hebdo attacks: lessons from the military milieu. Am J Emerg Med 33:843

6. Lockey DJ (2012) The shootings in Oslo and Utøya island July 22, 2011: Lessons for the International EMS community. Scand J Trauma Resusc Emerg Med 20:4 